\title{
Summation of blood glucose and TAG to characterise the "metabolic load index'
}

\author{
Sam R. Emerson ${ }^{1}$, Mark D. Haub ${ }^{1}$, Colby S. Teeman ${ }^{1}$, Stephanie P. Kurti ${ }^{2}$ and Sara K. Rosenkranz ${ }^{1 *}$ \\ ${ }^{1}$ Department of Food, Nutrition, Dietetics and Health, Kansas State University, 212 Justin Hall, 1324 Lovers Lane, Manhattan, \\ KS 66506, USA \\ ${ }^{2}$ Department of Kinesiology, Kansas State University, 1A Natatorium, Manhattan, KS 66506, USA
}

(Submitted 9 May 2016 - Final revision received 19 August 2016 - Accepted 10 September 2016 - First published online 24 October 2016)

\section{Abstract}

Research points to postprandial glucose and TAG measures as preferable assessments of cardiovascular risk as compared with fasting values. Although elevated postprandial glycaemic and lipaemic responses are thought to substantially increase chronic disease risk, postprandial glycaemia and lipaemia have historically only been considered separately. However, carbohydrates and fats can generally 'compete' for clearance from the stomach, small intestine, bloodstream and within the peripheral cell. Further, there are previous data demonstrating that the addition of carbohydrate to a high-fat meal blunts the postprandial lipaemic response, and the addition of fat to a high-carbohydrate meal blunts the postprandial glycaemic response. Thus, postprandial glycaemia and lipaemia are interrelated. The purpose of this brief review is 2-fold: first, to review the current evidence implicating postprandial glycaemia and lipaemia in chronic disease risk, and, second, to examine the possible utility of a single postprandial glycaemic and lipaemic summative value, which will be referred to as the metabolic load index. The potential benefits of the metabolic load index extend to the clinician, patient and researcher.

Key words: Lipaemia: Glycaemia: CVD: Metabolism: Circulating energy

Meal consumption, particularly meals that are high in fat, processed sugars and total energy content, results in transient elevations in blood glucose and lipids. These elevations, termed postprandial glycaemia and lipaemia, respectively, have both been linked to the progression of CVD, primarily through a resultant rise in oxidative stress, inflammation and endothelial dysfunction $^{(1,2)}$. Thus, exaggerated postprandial glycaemia and lipaemia, if experienced several times per day, represent a clear metabolic challenge to homoeostasis. Individuals in Western society are thought to spend most of their waking hours in the postprandial state ${ }^{(3)}$, and thus in a near-constant state of postprandial challenge, which could be amplified to prolonged dysmetabolism in the case of individuals at risk for metabolic disease. Hence, recent research has been devoted to better understand how the body, in health and disease, handles these metabolic excursions. Typically, postprandial glycaemic and lipaemic responses are characterised by measuring blood glucose and TAG serially for a given amount of time after a meal and, from these data, calculating AUC. Although these methods are effective in characterising the glycaemic and lipaemic responses independently, previous efforts have not considered them simultaneously. However, meals typically contain both carbohydrate and fat, as well as some protein; thus, it may be worthwhile to consider the summative metabolic response of both carbohydrate and lipids, as opposed to simply focusing on just one or the other. This brief review will first consider postprandial glycaemia and lipaemia independently in relation to metabolic status and CVD risk, followed by a discussion of the potential utility of a postprandial metabolic load index (MLI) that combines glycaemia and lipaemia together, indicating total circulating energy availability.

\section{Blood glucose and metabolic status/risk Fasting blood glucose, glycated $\mathrm{Hb}$ and cardiovascular risk}

Assessment of long-term glucose control via glycated $\mathrm{Hb}$ (HbA1c) or fasting glucose levels is commonly used as a valuation of cardiovascular and diabetic risk ${ }^{(4)}$. This practice is based on several epidemiological studies linking HbA1c levels or impaired fasting glucose to heart or vascular disease ${ }^{(5,6)}$. For example, the United Kingdom Prospective Diabetes Study found that mean HbA1c over time in the cohort was a valuable predictor of ischaemic heart disease ${ }^{(5)}$. Specifically, the data showed that for every $1 \%$ increase in HbA1c there was an approximately $10 \%$ increase in heart disease risk $^{(5)}$. With regard to fasting glucose levels, a meta-analysis of nearly 700000 people found that fasting blood glucose was modestly and

Abbreviations: A-MLI, adjusted metabolic load index; HbA1c, glycated Hb; MLI, metabolic load index; OGTT, oral glucose tolerance test.

* Corresponding author: S. K. Rosenkranz, fax +1 785532 3132, email SaraRose@ksu.edu 
non-linearly associated with vascular disease in diabetic individuals $^{(6)}$. Finally, an epidemiological study in US adolescents also found a link between impaired fasting glucose and CVD risk factors, including high fasting insulin, total and LDL-cholesterol, TAG, systolic blood pressure and low HDL-cholesterol $^{(7)}$. Thus, fasting glucose and HbA1c measures are considered by some to be valuable screening tools for type 2 diabetes and indeed are commonly used in clinical environments. However, it is interesting to note that early in the development of type 2 diabetes postprandial glucose tolerance plays a large role, whereas fasting glucose plays a more substantial role later in the disease process $^{(8)}$. In other words, derangements in fasting blood glucose take longer to develop, whereas impairments in post-challenge glucose tolerance could be observed earlier.

\section{Post-challenge/postprandial blood glucose and cardiovascular risk: epidemiological studies}

In contrast to impaired fasting glucose, there are substantial data suggesting that impaired glucose tolerance should be preferentially considered when assessing chronic disease risk $^{(9,10)}$. To support this, Tominaga et $a l .{ }^{(9)}$ found that survival rates in frank diabetes and impaired glucose tolerance were significantly lower than individuals with impaired fasting glucose, and further concluded that impaired glucose tolerance was a risk factor for CVD, but impaired fasting glucose was not. This finding was also supported by Blake et al. ${ }^{(10)}$ who followed 937 non-diabetic individuals for nearly 10 years and found that impaired glucose tolerance was phenotypically different from impaired fasting glucose, and is associated with increased presence of CVD risk factors. Further, the authors reported that individuals with impaired fasting glucose had CVD risk factors that were similar to individuals with normal glucose tolerance, further weakening the apparent utility of fasting glucose as a measure of CVD risk ${ }^{(10)}$.

A common way to assess glucose tolerance is the oral glucose tolerance test (OGTT), in which a plasma glucose measurement is made $2 \mathrm{~h}$ after the ingestion of a standard glucose load, typically 75 or $100 \mathrm{~g}^{(11)}$. There are numerous studies linking 2-h glucose levels following an OGTT with CVD risk ${ }^{(12-14)}$. The Chicago Heart Study found that 2 -h post-challenge glucose predicted all-cause mortality in a cohort of 11554 white men and 666 black men ${ }^{(13)}$. Similarly, results from the DECODE (Diabetes Epidemiology: Collaborative analysis of Diagnostic criteria in Europe) study indicated that high blood glucose following an OGTT was associated with an increased risk of death, independent of fasting glucose ${ }^{(14)}$. Finally, the Helsinki Policeman Study, Paris Prospective Study and the Whitehall Study all found that all-cause and CHD mortality was predicted by 2 -h post-challenge glucose following an OGTT ${ }^{(15-18)}$.

There are certainly drawbacks to the use of the OGTT to assess glucose tolerance and subsequent CVD risk. Consumption of $75-100 \mathrm{~g}$ of pure glucose rarely happens in daily life, and thus it does not perfectly simulate the real-life glucose challenges faced daily by many people. Further, the extent of glycaemia experienced following a meal is dependent on the entire contents of the test meal challenge. Hence, to better understand how the body tolerates glucose, it is important to challenge the metabolic system with a test meal that is representative of that experienced during daily living, such as a mixed meal. For this reason, some studies have utilised test meals that include fat and protein when assessing the glycaemic response and subsequent CVD risk ${ }^{(19,20)}$. Similar to studies using OGTT, the Diabetes Intervention Study found that the postprandial glycaemic response to a mixed meal was a significant predictor of $\mathrm{CHD}^{(19)}$. There is evidence that the glucose response seen following an OGTT is very similar to a mixed meal (in this case, consisting of wafers, rapeseed oil and honey), which suggests that the OGTT may have utility in assessing glucose tolerance, despite its apparent drawbacks ${ }^{(20)}$. Thus, the glucose response to an OGTT may potentially be used as a proxy for, but is not identical to, true-to-life postprandial glycaemia ${ }^{(2)}$.

\section{Post-challenge/postprandial blood glucose and cardiovascular risk: intervention studies}

An important intervention study connecting postprandial/postchallenge hyperglycaemia to CVD risk is the STOP-NIDDM (Study to Prevent Non-Insulin Dependent Diabetes Mellitus) trial $^{(21)}$. Results from the STOP-NIDDM trial show that treatment of individuals with impaired glucose tolerance with acarbose, an $\alpha$-glucosidase inhibitor that specifically reduces post-challenge glycaemia, is associated with a substantial (36\%) reduction in the likelihood of progressing to overt diabetes ${ }^{(21)}$. In addition, acarbose treatment was associated with a $34 \%$ risk reduction of developing hypertension and a $49 \%$ risk reduction for developing cardiovascular events ${ }^{(21)}$. Finally, in a subgroup of participants in which carotid intima media thickness was measured, treatment with acarbose was associated with a significantly attenuated progression of intima media thickness compared with the control group ${ }^{(22)}$. This work was subsequently supported by a meta-analysis that indicated a significant reduction in cardiovascular events following acarbose treatment in diabetic individuals, even after consideration of other risk factors ${ }^{(23)}$.

Another important intervention study by Esposito et al. ${ }^{(24)}$ investigated the effects of two insulin secretagogues (compounds that promote insulin secretion) repaglinide and glyburide, which have differing effectiveness on hyperglycaemia (with repaglinide being more effective), on surrogate measures of atherosclerosis. After 1 year, $52 \%$ of diabetic patients receiving repaglinide and $18 \%$ of participants receiving glyburide displayed carotid intima media thickness regression ${ }^{(24)}$. Further, C-reactive protein and IL-6, both markers of systemic inflammation, decreased more in the repaglinide group than in the glyburide group ${ }^{(24)}$. These findings suggest a direct link between postprandial/post-challenge glucose intolerance and CVD.

\section{Mechanisms behind blood glucose and cardiovascular risk}

How may post-challenge hyperglycaemia and CVD be mechanistically linked? First and foremost, glucose intolerance is typically a feature of insulin resistance ${ }^{(25)}$. Insulin resistance may result in deterioration of metabolic homoeostasis in terms of both glucose and circulating $\mathrm{NEFA}^{(25)}$. Not only do type 2 
diabetes patients experience impaired clearance of glucose by insulin they are also resistant to insulin-induced suppression of NEFA release ${ }^{(26)}$. If not controlled, the elevated NEFA levels will precipitate elevated hepatic glucose production ${ }^{(27)}$. The increased hepatic glucose production in combination with reduced insulinstimulated glucose uptake produces a spike in blood glucose levels ${ }^{(25)}$. The elevation of both fasting and postprandial glycaemia results in numerous adverse outcomes (Fig. 1).

To start, diabetic individuals typically experience endothelial dysfunction early in the disease process ${ }^{(28)}$. Specifically, the vasodilatory response is not only diminished in diabetics but is also related to glycaemic control ${ }^{(28)}$, which is supported by findings from in vivo studies demonstrating that hyperglycaemic spikes induce endothelial dysfunction in both normal and diabetic subjects ${ }^{(29,30)}$. Similarly, there are data showing that during the postprandial period in diabetic participants decreased flow-mediated dilation is negatively associated with the extent of postprandial glycaemia ${ }^{(31)}$. In addition, rapid upsurges of glucose result in non-reversible glycosylation of proteins ${ }^{(32)}$. These advanced glycosylated end products bind to endothelial smooth muscle receptors, precipitating increased endothelial permeability and vascular smooth muscle cell proliferation $^{(2,32)}$.

Additional avenues by which postprandial hyperglycaemia may be mechanistically linked to CVD are increased blood coagulation and/or thrombosis. When hyperglycaemia is induced experimentally, there is an increase in platelet aggregation ${ }^{(33)}$, fragments of prothrombin ${ }^{(34)}$ and a shortening of the half-life of fibrinogen ${ }^{(35)}$. There also may be an immune and inflammatory component to postprandial hyperglycaemia. Increases in TNF- $\alpha$ and IL- 6 have been shown to occur both in the context of postprandial hyperglycaemia ${ }^{(36)}$ and with a hyperglycaemic clamp ${ }^{(37)}$. In addition, circulating levels of intracellular adhesion molecule-1 (ICAM-1) have been demonstrated to increase substantially following post-challenge glycaemia, suggesting activation of one of the most proximal steps of the atheromatous lesion process ${ }^{(38)}$. Finally, LDL oxidation is known to increase in type 2 diabetic patients after meal consumption and is inversely proportional to the degree of metabolic control ${ }^{(39,40)}$. Collectively, these findings provide support for the notion of direct metabolic and physiologic links between postprandial/post-challenge hyperglycaemia and CVD risk.

\section{TAG and metabolic status/risk}

\section{Fasting TAG and CVD risk}

Similar to fasting blood glucose, elevated fasting TAG levels are typically thought to be a risk factor for CVD. However, the data supporting this relationship are somewhat equivocal. It appears that, unlike HDL-cholesterol and LDL-cholesterol for which there are strong, consistent relationships with cardiovascular risk $^{(41)}$, the data do not clearly reveal fasting TAG to be an independent risk factor for CVD. For example, the relationship

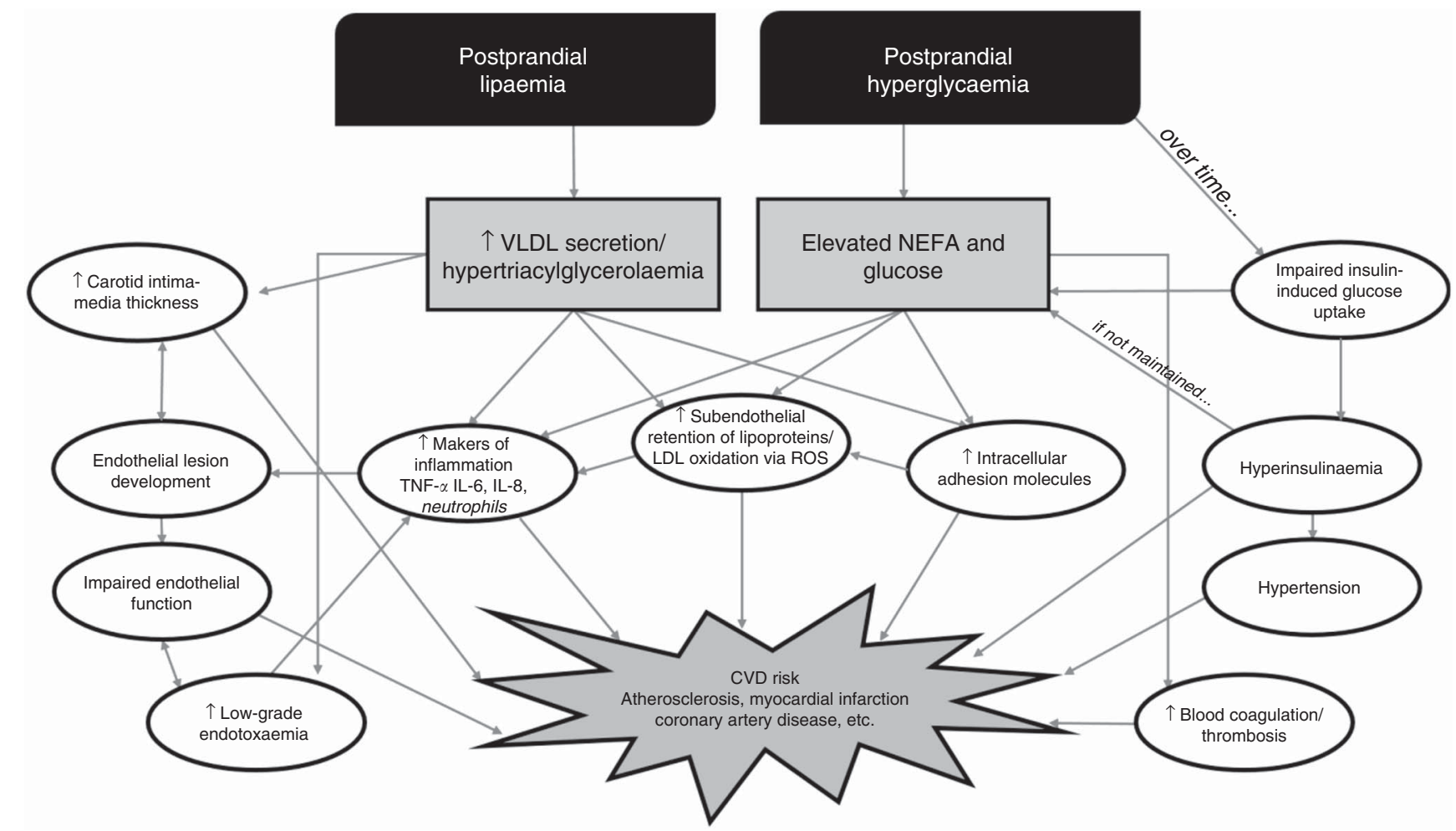

Fig. 1. Postprandial lipaemia, postprandial hyperglycaemia and CVD risk. Multiple potential mechanisms exist to explain the connection between postprandial dysmetabolism (glycaemia and lipaemia) and CVD risk. Similar primary facets involved in both postprandial glycaemia and lipaemia are elevated markers of systemic inflammation, increased subendothelial retention of lipoproteins and increased intracellular adhesion molecules. See the 'Mechanisms behind blood glucose and cardiovascular risk' and 'Mechanisms behind TAG and cardiovascular risk' sections for more details. ROS, reactive oxygen species. 
between TAG levels and cardiovascular risk, although significant in univariate analyses, sometimes disappears or weakens in multivariate analyses that control for HDLcholesterol $^{(42)}$. In addition, measurement of TAG, clinically or experimentally, can fluctuate greatly based on recent dietary composition, physical activity or weight status ${ }^{(42,43)}$. However, some evidence does point to the utility of measuring fasting TAG in order to assess cardiovascular risk ${ }^{(44,45)}$. First, the Copenhagen Male Study, which featured an 8-year follow-up of nearly 3000 middle- to older-aged men, found that men in the middle and highest tertile groups of fasting TAG levels had an increased risk for ischaemic heart disease, as well as a risk gradient based on TAG levels, even after being stratified for HDLcholesterol $^{(44)}$. Intriguingly, the middle and highest tertiles of TAG levels displayed relative risks for ischaemic heart disease of 1.5 and $2 \cdot 2$, respectively, compared with the lowest tertile, even after consideration of LDL-cholesterol, HDL-cholesterol, physical activity, hypertension, type 2 diabetes, smoking, BMI and age ${ }^{(44)}$. Further, a meta-analysis that included 46413 men and 10864 women across seventeen population-based, prospective studies found that elevated TAG levels were associated with an approximately $30 \%$ increase in CVD risk in men and an approximately $75 \%$ increase in women ${ }^{(45)}$. Although controlling for HDL-cholesterol and LDL-cholesterol indicated a lowered associated risk between TAG and CVD, having elevated TAG levels was still a statistically significant risk factor.

\section{Postprandial TAG and CVD risk}

Although the clinical and experimental utility of measuring fasting TAG remains equivocal, there is substantial evidence suggesting that the transient, extensive rise in blood lipids (namely, TAG) following a meal can have direct atherosclerotic effects $^{(46)}$. Thus, postprandial lipaemia is considered by many to be an independent CVD risk factor ${ }^{(2,47)}$. First, whereas the relationship between fasting TAG levels and cardiovascular risk can be confounded by HDL-cholesterol levels ${ }^{(42)}$, there are data demonstrating that as TAG increase in the postprandial period there is a related decrease in certain HDL-cholesterol subfractions $^{(48)}$. Furthermore, postprandial levels of certain TAG-rich lipoprotein subfractions (particularly, small chylomicron remnants) were found to be correlated with coronary lesion progression in thirty-two post-infarction men, even after controlling for HDL-cholesterol and dense LDL apo $\mathrm{B}^{(49)}$. In a landmark study by Patsch et $a l .{ }^{(46)}$, forty control subjects and sixty-one subjects with severe coronary artery disease (CAD) completed meal tolerance tests in order to assess postprandial lipaemia and CAD risk. Measures of postprandial lipaemia (peak and AUC values) were significantly greater in the CAD patients than in the controls ${ }^{(46)}$. Furthermore, postprandial lipaemic values predicted the presence or absence of CAD with $68 \%$ accuracy by logistic regression analysis ${ }^{(46)}$. Although not synonymous with postprandial TAG, non-fasting TAG still provide a way to examine the blood lipid levels that a person may experience in daily life, and are more practical for large-scale studies. In a prospective study of 26509 initially healthy women, fasting and non-fasting TAG were assessed over approximately 11 years of follow-up, and hazard ratios for incident cardiovascular events were determined ${ }^{(50)}$. Although both baseline fasting and non-fasting TAG levels correlated with cardiac risk factors and markers of insulin resistance, the relationship disappeared with regard to fasting TAG values after covarying total cholesterol, HDL-cholesterol and measures of insulin resistance ${ }^{(50)}$. On the other hand, the relationship between non-fasting TAG and cardiac events remained robust even in the fully adjusted model (increasing tertiles of non-fasting TAG levels: 1 (reference tertile), 1.44 and 1.98), thus supporting the use of non-fasting (or postprandial) TAG measures over fasting TAG measures when predicting cardiovascular risk $^{(50)}$.

\section{Mechanisms behind TAG and cardiovascular risk}

The primary mechanism explaining how elevated TAG levels, be it fasting, non-fasting or postprandial, induce CVD is the subendothelial retention of lipoproteins, which initiates atherogenesis $^{(51)}$. Circulating chylomicrons and large VLDL molecules, which are rich in TAG, are too big to penetrate the arterial wall, but smaller dense VLDL and other remnant molecules can enter the arterial wall and bind to proteoglycans $^{(52)}$. The presence of these lipoprotein and remnant molecules, which can be elevated during acute postprandial lipaemia, initiates a local inflammatory response that includes macrophages and $\mathrm{T}$ cells that promote subsequent lesion development ${ }^{(51)}$. This hypothesis is in line with studies demonstrating that the extent of coronary blockage and subsequent CVD risk is associated with the degree of postprandial lipaemia following a high-fat meal ${ }^{(46,49)}$. In addition, there are also data suggesting that carotid intima media thickness is significantly positively associated with the postprandial lipaemic response in both healthy and diabetic individuals ${ }^{(53,54)}$. Further, many studies have provided evidence suggesting that markers of inflammation significantly increase in the presence of postprandial lipaemia, including plasma IL-8, neutrophil counts, ICAM-1 and TNF- $\alpha^{(36,55,56)}$.

Another avenue by which lipaemia may increase cardiovascular risk is impaired endothelial function. It has been shown that endothelial function, measured as brachial artery blood flow, can be impaired in response to a high-fat meal, providing a potential mechanistic link between postprandial lipaemia and heart disease, independent of cholesterol ${ }^{(57)}$. Interestingly, a study by Erridge et $a l^{(58)}$ found a significant increase in circulating bacterial endotoxin following a high-fat meal, suggesting that low-grade endotoxaemia may contribute to the inflammatory state that occurs after a high-fat meal, and may explain the endothelial dysfunction that occurs during postprandial lipaemia. As a whole, evidence suggests several overlapping mechanisms by which elevated TAG levels, fasting or postprandial, may lead to increased CVD risk (Fig. 1).

\section{Similarities between glycaemia and lipaemia}

As postprandial glucose and lipids both appear to fluctuate from homeostasis on a daily basis in normal living, and more importantly these aberrant responses appear to be strongly 
linked to cardiovascular risk, it may be worth considering them in unison, compared with independently as is currently common practice. However, in order to understand the ways in which glycaemia and lipaemia are linked, it is vital to discuss and recognise the overlapping metabolic pathways shared between dietary carbohydrate and lipids.

\section{Uptake into peripheral cells}

Circulating blood glucose is taken up by human cells via facilitated diffusion across the plasma membrane. There are at least six known GLUT that perform this task ${ }^{(59)}$. GLUT4 is the most widely acknowledged, as it is expressed on adipose cells, skeletal muscle cells, cardiac muscle cells and other insulinsensitive cells ${ }^{(60)}$. As insulin binds to its receptor, GLUT4 is translocated to the plasma membrane and glucose enters the cell ${ }^{(60)}$.

There are two primary ways in which lipid (specifically, fatty acids) can become available for energy use in the cytosol of a given cell. One option is for fatty acids to diffuse across the plasma membrane of the cell, similar to carbohydrate. These incoming fatty acids are either: (1) freed from TAG in lipoproteins by the action of lipoprotein lipase (LPL); (2) circulating bound to albumin; or (3) circulating as NEFA. The other option for fatty acids to become available in the cytosol is liberation from intracellular lipid pools. Regardless of how they arrive in the cytosol, fatty acids are typically found linked to fatty acid binding protein ${ }^{(61)}$.

In discussing the uptake of fat and carbohydrate into peripheral cells, it is worth considering the shared relationship of both glucose and fatty acids with the hormone insulin. As discussed previously (see the 'Mechanisms behind blood glucose and cardiovascular risk' section), insulin works to both allow entrance of glucose into peripheral cells and suppress the release of NEFA into circulation. Thus, insulin clearly plays a major role in the regulation of circulating glucose and fatty acids. Further, as elevations in circulating NEFA can precipitate augmented hepatic glucose production ${ }^{(27)}$, perturbations in the level of one substrate can affect the other. Therefore, based on the data there is a relationship between an individual's circulating levels of insulin, glucose and fatty acids, and consequently derangement of one substrate or mechanism (e.g. insulin sensitivity) can produce a downward metabolic spiral $^{(25)}$.

Another relevant consideration when discussing uptake of carbohydrate and lipid is the effect of dietary fats on insulin resistance. Evidence suggests that increased levels of fatty acids, both in the diet and in circulation, can play a role in the development of insulin resistance ${ }^{(62)}$. The level of fatty acids in the diet and circulation, as well as a the type, that is the proportion of SFA relative to unsaturated fatty acids, will be reflected in the fatty acid composition of peripheral cell membranes $^{(63)}$. Higher levels of SFA in cell membranes appear to substantially hinder insulin action ${ }^{(62)}$. In addition, as Randle et $a l .{ }^{(64)}$ first suggested in 1963 , elevated fatty acids are likely to compete with glucose for clearance via oxidation, resulting in elevated blood glucose, and thus high fatty acid intake can be directly responsible for the body's inability to clear glucose.
Over time this could lead to severely impaired glucose disposal and overt diabetes. It also appears that high levels of myocellular lipid can impair insulin sensitivity ${ }^{(65,66)}$. As the traditional Western diet is typically high in fat, especially SFA, these findings represent additional ways in which dietary lipid and carbohydrate intake are interconnected in the determination of chronic disease risk.

\section{Major metabolic pathways}

The initial step towards obtaining energy from glucose present in the cytosol is glycolysis, followed by the Kreb's cycle and the electron transport chain ${ }^{(67,68)}$. Glycolysis is a set of ten reactions by which glucose is converted to two molecules of a pyruvate, with two ATP molecules and one hydrogenated NADH molecule produced in the process. The newly generated pyruvate can have one of several different destinations, including entering the mitochondria and being converted into acetyl CoA via pyruvate dehydrogenase, or being converted to alanine or other amino acids, lactic acid, oxaloacetate or back to glucose.

In order to obtain energy from the fatty acids located in the cytosol, the final destination is the Kreb's cycle as well, although there is a longer and more complex path. The process of fatty acid oxidation, known as $\beta$-oxidation, is a four-step process that results in the products $\mathrm{FADH} 2, \mathrm{NADH}+\mathrm{H}^{+}$and acetyl $\mathrm{CoA}$ being produced. The acetyl CoA produced via $\beta$-oxidation can either travel to the liver to form ketone bodies or condense with oxaloacetate to form citrate and enter the citric acid cycle. (It should be noted that odd-chain fatty acids and unsaturated fatty acids require additional steps and/or enzymes compared with even-chain SFA, which were discussed here for simplicity.)

Acetyl CoA, whether its original source was lipid, carbohydrate or otherwise, enters the citric acid cycle, also known as the Kreb's cycle, where it condenses with oxaloacetate to form citrate. Eight subsequent reactions occur, after which oxaloacetate is again present, thus making the process cyclic. During one round of the citric acid cycle, two carbon dioxide molecules, three $\mathrm{NADH}^{+}$molecules, one FADH2 molecule and one ATP molecule are generated. $\mathrm{NADH}^{+}$and $\mathrm{FADH} 2$ can then transfer their electrons to the electron transport chain, through which oxidative phosphorylation produces more ATP.

\section{An overwhelmed pathway}

It is important to consider the postprandial glycaemic and lipaemic responses together because they ultimately share similar destinations or outlets. Although details vary depending on the physiological site, carbohydrates and fats can generally 'compete' for clearance from the stomach, small intestine, bloodstream and within the peripheral cell. Specifically with regard to the latter, both carbohydrates and lipids are largely converted to acetyl CoA and enter the citric acid cycle and the electron transport chain, as previously discussed. Consumption of large-volume, energy-dense and easily digestible food precipitates substantial spikes in NEFA, TAG and blood glucose. As the fat and carbohydrate compete for clearance, the excessive upsurge of metabolic energy overwhelms the metabolic capacity of the mitochondria within adipose and 
skeletal muscle cells, as well as all other metabolically active cells ${ }^{(69)}$. Fatty acids and glucose swamp the citric acid cycle, which promotes the excess production of $\mathrm{NADH}^{+}$that outperforms the electron transport chain ${ }^{(2)}$. The buildup of $\mathrm{NADH}^{+}$ raises the proton gradient within the mitochondria, promoting the transfer of single electrons to $\mathrm{O}_{2}$, generating free radicals that include the superoxide anion ${ }^{(2,70,71)}$. The elevated production of reactive oxygen species will subsequently oxidise LDL particles that have penetrated the endothelium and entered the subendothelial space ${ }^{(72,73)}$. Oxidised LDL particles lead to an inflammatory cascade in the following ways: (1) increased expression of cellular adhesion molecules, which promote attachment and penetration of immune cells to the endothelium $^{(74)}$; (2) elevated release of pro-inflammatory cytokines $^{(75)}$; (3) activation of endothelial and smooth muscle cells ${ }^{(76)}$; and (4) increased uptake of the oxidised LDL particles by M2 macophages, triggering additional release of pro-inflammatory cytokines ${ }^{(73)}$. Thus, the subendothelial penetration of lipoproteins and their subsequent oxidation by reactive oxygen species - both downstream results of the energy excess in circulation - can precipitate increased inflammation, sympathetic tone and vasoconstriction, as well as thrombogenicity and advanced glycation end products - all of which can increase an individual's CVD risk ${ }^{(2,69,77,78)}$. Therefore, as both glucose and fatty acids can 'team up' to overwhelm the metabolic machinery of the body and produce the above response, it does not seem unreasonable to consider the glycaemic and lipaemic responses in the postprandial period together.

\section{Summing glucose and TAG to characterise 'metabolic load index'}

Given the potential utility and value in assessing postprandial glycaemia and lipaemia in unison, we suggest a summative index that we have termed 'metabolic load index'. To create an MLI score, the clinician or scientist could simply combine the values of circulating blood glucose and TAG:

$$
\operatorname{MLI}\left(\frac{\mathrm{mg}}{\mathrm{dl}}\right)=\operatorname{TAG}\left(\frac{\mathrm{mg}}{\mathrm{dl}}\right)+\text { Glu }\left(\frac{\mathrm{mg}}{\mathrm{dl}}\right) \text {. }
$$

For instance, if considering a single time point, glucose and TAG could be summed to determine the MLI in $\mathrm{mg} / \mathrm{dl}$ for that point in time. The principle would remain the same if AUC was being considered: simply sum the TAG and glucose AUC values. In this way, the researcher and clinician could get an idea of the overall metabolic load, or challenge, that the patient or participant is experiencing, as well as the volume of circulating available energy, either at that specific time point or over time (such as following a meal).

Fig. 2 demonstrates MLI utilising published data from our laboratory, which displays hourly changes in glucose, TAG and MLI following a high-fat meal ${ }^{(79)}$. The test meal consisted of ice cream and whipping cream $(1 \mathrm{~g} / \mathrm{kg}$ body weight carbohydrate and $1 \mathrm{~g} / \mathrm{kg}$ body weight fat, approximately $60 \%$ fat, $20 \%$ carbohydrate). MLI is the sum of TAG and glucose at each time point. It is interesting to note that, as the meal contained approximately $60 \%$ fat, the MLI closely follows the change in TAG over time, most likely because of the large contribution of

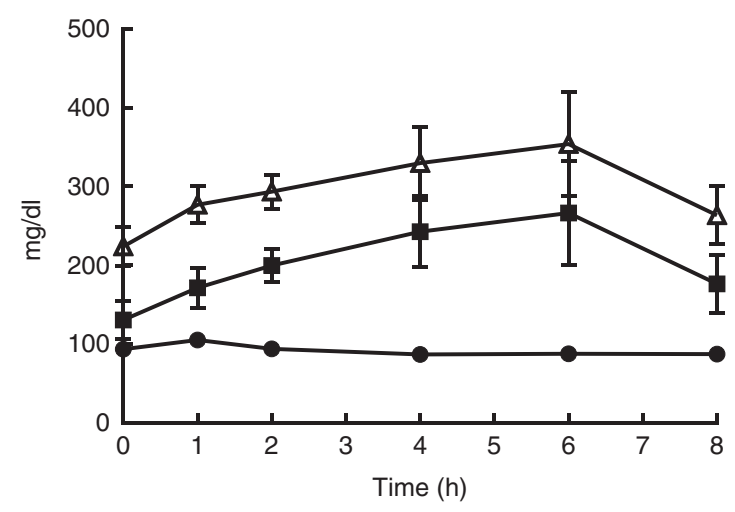

Fig. 2. Hourly changes in glucose, TAG and metabolic load index following a high-fat meal. These data were taken from a previously published study in our laboratory $^{(79)}$. This figure displays glucose (0),TAG (ם) and metabolic load index $(\Delta)$ at baseline (time 0 ) and for $8 \mathrm{~h}$ during the postprandial period following consumption of a high-fat meal. With these data, fasting and postprandial metabolic load index can be determined by adding the TAG and glucose value at each time point. See the 'Summing glucose and TAG to characterise "metabolic load index"' section for more details. Values are means, with their standard errors.

fat to the total energy content of the meal. Similarly, as the carbohydrate load in the meal was relatively low, the blood glucose response was marginal and negligibly affected the MLI during the postprandial period. This test meal was high in fat, and few people would likely deem the meal 'healthy'. However, if blood glucose response was the main/only outcome of interest, the conclusion after consumption of such a meal could be that metabolic disturbance was minimal, although this is clearly not the case when the TAG and MLI responses are considered together. Thus, clinically, assessment of MLI could provide a measurement of metabolic challenge or disturbance without biasing towards glucose or TAG alone. Further, consideration of MLI may be more relevant when determining metabolic responses to mixed meals that are more typically consumed in daily living, as compared with an all-lipid or all-carbohydrate meal, as is used in an OGTT.

To support this notion, there is evidence indicating that postprandial fat and carbohydrate metabolism are not independent processes, and alterations in one can affect the other. An early study elucidating this relationship was conducted by Albrink et al. ${ }^{(80)}$ in 1958 in which a 60-g fat meal was consumed with and without $100-250 \mathrm{~g}$ of added glucose. The authors found that the addition of glucose either diminished or completely abolished the postprandial rise in TAG, depending on the amount of added glucose ${ }^{(80)}$. In a related study several years later, Mann et $a l^{(81)}$ found that postprandial lipaemia was cleared more slowly following a meal that contained a glucose load compared with a meal that contained sucrose. These early findings have been supported by several more recent studies demonstrating similar results ${ }^{(82-84)}$. In a randomised cross-over study, Westphal et al. ${ }^{\left({ }^{84)}\right.}$ investigated the effect of adding $75 \mathrm{~g}$ of glucose to a high-fat meal compared with a high-fat meal without the added glucose on TAG, VLDL and chylomicrons (Fig. 3). The addition of glucose to the meal resulted in a delay in the TAG response, as well as a lower peak ${ }^{(84)}$. There was also a $42 \%$ reduction in incremental AUC for the meal with glucose 


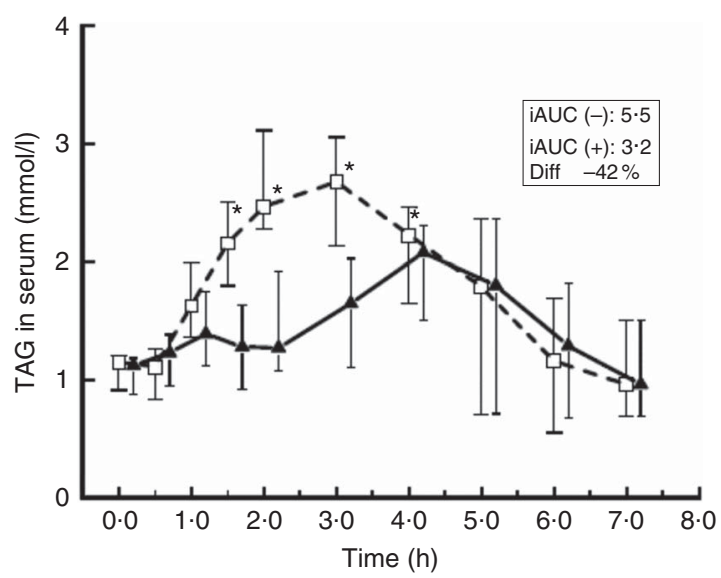

Fig. 3. Postprandial TAG responses to a high-fat meal with $(\mathbf{A})$ and without ( $\square$ ) $75 \mathrm{~g}$ of added glucose. The TAG response is blunted in terms of both peak value and time to peak when glucose is added to the high-fat meal. Incremental AUC (iAUC) is $42 \%$ lower in the fat meal with added glucose compared with the fat meal alone $(P=0.017)$. ${ }^{*}$ Mean value was significantly different from that of the meal with added glucose at the same time point $(P<0.05)$. Adapted with permission from Westphal et al. ${ }^{(84)}$. Diff, difference.

compared with the fatty meal alone. Finally, there was a delayed chylomicron response and a blunted VLDL response. A similar investigation by Knuth et $a l .{ }^{(83)}$ demonstrated a reduction in postprandial plasma TAG in women following a fat meal containing additional carbohydrate compared with a fat meal alone. Collectively, these studies suggest that the addition of carbohydrate to a meal and the resultant glycaemic response alters the postprandial rise in TAG. The interrelationship between postprandial glycaemia and lipaemia also holds when adding fat to a high-carbohydrate meal ${ }^{(85,86)}$. In an investigation by Collier \& O'Dea ${ }^{(85)}$, when $50 \mathrm{~g}$ of fat (as butter) was added to $50 \mathrm{~g}$ of carbohydrate (as potato), the postprandial glucose response was significantly blunted compared with ingestion of potato alone. This mitigation was likely a result of delayed glucose absorption in the small intestine, consequent to a fat-induced repression of gastric emptying. Together, these findings suggest that postprandial glucose and lipid metabolism are not independent processes - rather they are interrelated, and consequently it would be valuable to consider them together.

There may also be merit in considering an energy-adjusted version of the MLI. Lipid typically yields approximately $38 \mathrm{~kJ} / \mathrm{g}$ $(9 \mathrm{kcal} / \mathrm{g})$ and carbohydrate yields approximately $17 \mathrm{~kJ} / \mathrm{g}$ ( $4 \mathrm{kcal} / \mathrm{g}$ ). In an attempt to consider the amount of circulating energy in the bloodstream at a given time, there may be utility in adjusting the MLI equation to reflect the difference in energy yield between carbohydrate and fat, which could be accomplished via an 'adjusted metabolic load index' (A-MLI):

$$
\begin{aligned}
\operatorname{A-MLI}\left(\frac{\mathrm{kJ}}{\mathrm{dl}}\right) & =\left(\operatorname{TAG}\left(\frac{\mathrm{mg}}{\mathrm{dl}}\right) \times 0.001 \frac{\mathrm{g}}{\mathrm{mg}} \times 38 \frac{\mathrm{kJ}}{\mathrm{g}}\right) \\
& +\left(\operatorname{Glu}\left(\frac{\mathrm{mg}}{\mathrm{dl}}\right) \times 0.001 \frac{\mathrm{g}}{\mathrm{mg}} \times 17 \frac{\mathrm{kJ}}{\mathrm{g}}\right) .
\end{aligned}
$$

For instance, if an individual was found to have TAG levels of $120 \mathrm{mg} / \mathrm{dl}$ and glucose levels of $90 \mathrm{mg} / \mathrm{dl}$, their A-MLI would be
$6.09 \mathrm{~kJ} / \mathrm{dl}(1.44 \mathrm{kcal} / \mathrm{dl})$. Thus, the A-MLI would provide an equation through which to quantify or estimate the available circulating energy from fat and carbohydrate. However, there are some issues with the A-MLI, which include biasing towards TAG and presenting somewhat unusual units $(\mathrm{kJ} / \mathrm{dl}(\mathrm{kcal} / \mathrm{dl}))$. Nevertheless, the A-MLI may prove to be a valuable clinical tool in assessing the metabolic state. Research comparing MLI and A-MLI with regard to prediction of, and association with, health outcomes would be valuable.

\section{Utility of the metabolic load index}

What are some ways or instances in which utilisation of the MLI would be beneficial or enhance our understanding of an individual's metabolic status? First and most clearly, use of the MLI could better reveal the degree of metabolic challenge that a person is experiencing either while fasting or after a meal. This utility would be particularly applicable to patient populations, as it has already been previously discussed how fluctuations in postprandial glycaemia and lipaemia can be cardiovascular risk factors. Specifically, it may be valuable to develop a 'cut point' for fasting MLI, such as $200 \mathrm{mg} / \mathrm{dl}$, as postprandial assessments may be too cumbersome in certain clinical settings. Particular patient populations for which assessing MLI would be valuable might include individuals with diagnosed CVD or type 2 diabetes. As metabolism of lipids and carbohydrates is so interrelated, consideration of the glycaemic or lipaemic response together would be valuable.

MLI would also be useful in terms of standardising responses across studies investigating postprandial metabolism. One of the most difficult aspects of compiling and comparing studies either investigating postprandial lipaemia or glycaemia (when a mixed meal is used) is the heterogeneity of test meal challenges that are utilised in the investigations. Although differences in the amount of total energy (and perhaps participants' body mass) would still need to be considered, MLI may help in comparing across studies that use meals of different macronutrient distribution but similar energetic value.

A current area of debate is the ideal proportion of macronutrients that should be consumed in a healthy diet. If the MLI response is similar between diets of different macronutrient distribution but similar energetic load, it may make the focus on select macronutrient profiles less prevalent.

\section{Considerations of the metabolic load index}

Given the novelty of the MLI, there are certainly considerations to be made. First, it has been shown that dietary fructose leads to postprandial metabolic responses that are different from dietary glucose $\mathrm{e}^{(87)}$. Specifically, fructose consumption (compared with glucose) as part of a mixed meal will lead to: (1) a greater TAG response, particularly in the latter stages of a postprandial assessment; (2) a comparatively negligible glucose response; and (3) a much more tempered insulin response ${ }^{(87)}$. However, we assert that the different metabolic responses of glucose and fructose do not represent a weakness of MLI, but rather a strength. Considering the different metabolic consequences of glucose and fructose, the outcomes of interest 
are nevertheless the same: circulating glucose and TAG, the two markers measured in the MLI. Thus, although fructose may lead to a greater TAG response and a lower glucose response compared with dietary glucose ${ }^{(87)}$, this will be captured in the MLI. If only considering the blood glucose response, one could surmise that fructose is better to include in a mixed meal because there is a much less blood glucose response. However, utilising the MLI, it can be seen that fructose still has substantial metabolic effects - though it more drastically alters the other component of the MLI equation, TAG. Thus, we assert that a strength of MLI is that it 'throws a broader net'. In other words, even though many nutrients clearly have differing metabolic effects such as glucose and fructose, MLI is better equipped than blood glucose or TAG assessment alone to capture the metabolic challenge that the body is experiencing.

It would be valuable to determine an optimal postprandial time point to assess MLI. For instance, it appears that TAG measured 2 and $4 \mathrm{~h}$ after meal consumption have the strongest association with cardiovascular events ${ }^{(50)}$ and that TAG tend to peak $4 \mathrm{~h}$ after a meal ${ }^{(88,89)}$. In addition, one study has found that measuring TAG $4 \mathrm{~h}$ post a high-fat meal is a suitable surrogate for longer and more involved postprandial lipaemia assessments ${ }^{(90)}$. On the other hand, as previously discussed, postchallenge glucose tolerance is typically assessed in the context of a 2 -h OGTT ${ }^{(11)}$, and 2 -h glucose values are strongly related to CVD risk ${ }^{(12-14)}$. Clearly, there are differences in the timing of postprandial glucose and TAG responses and, consequently, in the timing of assessment to best ascertain an individual's disease risk. Considering these differences, it is difficult to state an optimal, single time point at which to assess MLI in clinical and research settings. It would be very worthwhile for future studies to address this question using large data sets of postprandial metabolic markers and disease risk outcomes.

Similarly, a logical question is as follows: which individuals would benefit most from ascertaining their fasting and postprandial MLI? An expert panel statement has suggested that postprandial TAG assessments are most valuable in individuals with fasting levels between 89 and $180 \mathrm{mg} / \mathrm{dl}^{(91)}$. The rationale for this stance is that individuals with fasting TAG $<89 \mathrm{mg} / \mathrm{dl}$ will typically show a negligible postprandial response, whereas those with fasting TAG $>180 \mathrm{mg} / \mathrm{dl}$ will present an excessively delayed and exaggerated postprandial lipaemic response such that there is little diagnostic utility ${ }^{(91)}$. In line with this, there may be certain populations that could most benefit diagnostically from determination of MLI. It could be that individuals with very low or high fasting glucose and/or TAG levels may not benefit very much from the additional metabolic information provided via MLI. However, we are hesitant to state which individuals would and would not benefit from fasting and postprandial MLI assessment, given the current dearth of evidence to support a conclusion. Further, as addressed previously, with both glucose and TAG, postprandial values are typically better predictors of CVD risk compared with fasting values. Thus, there is no compelling evidence suggesting that individuals with certain fasting MLI values would not benefit substantially from a postprandial MLI assessment. It would be advantageous for future research to further explore this concept.
Finally, it is likely that genetics influences the postprandial MLI response. This supposition is based on the notion that gene variation has been found to contribute to inter-individual differences in both postprandial lipaemia and hyperglycaemia. With regard to postprandial lipaemia, the most heavily studied genetic regions have been the encoding of various apo genes, such as $A P O A 1^{(92)}$ and $A P O C 3^{(93)}$, and genes encoding lipid metabolism enzymes such as $L P L^{(94)}$ and $H L$ (hepatic lipase) ${ }^{(95)}$. A few areas of genetic contribution to hyperglycaemia include adiponectin gene polymorphisms ${ }^{(96,97)}$, telomere length ${ }^{(98)}$ and transcription factor 7-like 2 (TCF $7 L 2)$ variation $^{(99)}$, a transcription factor that is to date the most substantial genetic contributor to type 2 diabetes incidence ${ }^{(100)}$. Clearly, as there are established genetic contributors to both postprandial lipaemia and hyperglycaemia, it is logical to assume that genetic factors influence MLI as well.

\section{Conclusion and future directions}

It has been well established that postprandial glycaemia and lipaemia are risk factors for CVD. Specifically, postprandial glycaemia and lipaemia have been linked to increased disease risk by way of oxidative stress, inflammation and endothelial dysfunction, as well as other mechanisms. Although carbohydrate and lipids ultimately share similar clearing mechanisms, the fluctuations in these substrates in daily life, or experimentally following a test meal challenge, have historically only been considered separately. However, in light of evidence that postprandial glycaemia and lipaemia are not independent of one another, it does not seem unreasonable to consider both of these phenomena together in a single index. The concept of MLI, which is the sum of blood glucose and TAG, is valuable in that it considers the total metabolic challenge that the body is experiencing, either at a single time point or over time. In our opinion, the MLI carries significant potential clinical utility. It would be worthwhile for future investigations to assess MLI in the context of disease risk, investigating the relationship between MLI and markers of inflammation, oxidative stress, endothelial dysfunction and other risk markers. However, currently we do not know whether adding the two outcomes in a $1: 1$ ratio is the most accurate way to predict risk when considering glucose and TAG together. Thus, future work should seek to derive the best equation for assessing glycaemia and lipaemia simultaneously by using data from clinical populations and longitudinal studies.

\section{Acknowledgements}

The authors contributed to the completion of the present review in the following ways: S. R. E. helped develop the manuscript idea and wrote the manuscript, M. D. H. helped develop the manuscript idea and edited the manuscript, C. S. T. edited the manuscript and provided vital feedback, S. P. K. edited the manuscript and provided vital feedback and S. K. R. helped develop the manuscript idea and edited the manuscript.

This research received no specific grant from any funding agency, commercial or not-for-profit sectors.

The authors declare they have no conflicts of interest. 


\section{References}

1. Ceriello A (2005) Postprandial hyperglycemia and diabetes complications: is it time to treat? Diabetes 54, 1-7.

2. O'Keefe JH \& Bell DS (2007) Postprandial hyperglycemia/ hyperlipidemia (postprandial dysmetabolism) is a cardiovascular risk factor. Am J Cardiol 100, 899-904

3. Sies H, Stahl W \& Sevanian A (2005) Nutritional, dietary and postprandial oxidative stress. J Nutr 135, 969-972.

4. Alberti KG \& Zimmet PZ (1998) Definition, diagnosis and classification of diabetes mellitus and its complications. Part 1: diagnosis and classification of diabetes mellitus. Provisional report of a WHO consultation. Diabet Med 15, 539-553.

5. Stratton IM, Adler AI, Neil HA, et al. (2000) Association of glycaemia with macrovascular and microvascular complications of type 2 diabetes (UKPDS 35): prospective observational study. BMJ 321, 405-412.

6. Emerging Risk Factors Collaboration (2010) Diabetes mellitus, fasting blood glucose concentration, and risk of vascular disease: a collaborative meta-analysis of 102 prospective studies. Lancet 375, 2215-2222.

7. Williams DE, Cadwell BL, Cheng YJ, et al. (2005) Prevalence of impaired fasting glucose and its relationship with cardiovascular disease risk factors in US adolescents, 1999-2000. Pediatrics 116, 1122-1126.

8. Monnier L, Lapinski H \& Colette C (2003) Contributions of fasting and postprandial plasma glucose increments to the overall diurnal hyperglycemia of type 2 diabetic patients: variations with increasing levels of $\mathrm{HbA}(1 \mathrm{c})$. Diabetes Care 26, 881-885.

9. Tominaga M, Eguchi H, Manaka H, et al. (1999) Impaired glucose tolerance is a risk factor for cardiovascular disease, but not impaired fasting glucose. The Funagata Diabetes Study. Diabetes Care 22, 920-924.

10. Blake DR, Meigs JB, Muller DC, et al. (2004) Impaired glucose tolerance, but not impaired fasting glucose, is associated with increased levels of coronary heart disease risk factors: results from the Baltimore Longitudinal Study on Aging. Diabetes 53, 2095-2100.

11. Matsuda M \& DeFronzo RA (1999) Insulin sensitivity indices obtained from oral glucose tolerance testing: comparison with the euglycemic insulin clamp. Diabetes Care 22, 1462-1470.

12. Levitan EB, Song Y, Ford ES, et al. (2004) Is nondiabetic hyperglycemia a risk factor for cardiovascular disease?: a meta-analysis of prospective studies. Arch Intern Med $\mathbf{1 6 4}$ $2147-2155$.

13. Lowe LP, Liu K, Greenland P, et al. (1997) Diabetes, asymptomatic hyperglycemia, and 22-year mortality in black and white men. The Chicago Heart Association Detection Project in Industry Study. Diabetes Care 20, 163-169.

14. Pekkanen J, Tuomilehto J, Qiao Q, et al. (1999) Glucose tolerance and mortality: comparison of WHO and American Diabetes Association diagnostic criteria. Lancet 354, 617-621.

15. Balkau B, Shipley M, Jarrett RJ, et al. (1998) High blood glucose concentration is a risk factor for mortality in middleaged nondiabetic men. 20-year follow-up in the Whitehall Study, the Paris Prospective Study, and the Helsinki Policemen Study. Diabetes Care 21, 360-367.

16. Ducimetiere P, Eschwege E, Papoz L, et al. (1980) Relationship of plasma insulin levels to the incidence of myocardial infarction and coronary heart disease mortality in a middle-aged population. Diabetologia 19, 205-210.

17. Pyörälä K, Savolainen E, Lehtovirta E, et al. (1979) Glucose tolerance and coronary heart disease: Helsinki Policemen Study. J Chronic Dis 32, 729-745.
18. Reid D, Hamilton P, Keen H, et al. (1974) Cardiorespiratory disease and diabetes among middle-aged male civil servants: a study of screening and intervention. Lancet 303, 469-473.

19. Hanefeld M, Fischer S, Julius U, et al. (1996) Risk factors for myocardial infarction and death in newly detected NIDDM: the Diabetes Intervention Study, 11-year follow-up. Diabetologia 39, 1577-1583.

20. Wolever TM, Chiasson JL, Csima A, et al. (1998) Variation of postprandial plasma glucose, palatability, and symptoms associated with a standardized mixed test meal versus $75 \mathrm{~g}$ oral glucose. Diabetes Care 21, 336-340.

21. Chiasson J, Josse RG, Gomis R, et al. (2002) Acarbose for prevention of type 2 diabetes mellitus: the STOP-NIDDM randomised trial. Lancet 359, 2072-2077.

22. Chiasson J, Josse RG, Gomis R, et al. (2003) Acarbose treatment and the risk of cardiovascular disease and hypertension in patients with impaired glucose tolerance: the STOP-NIDDM trial. JAMA 290, 486-494.

23. Hanefeld M, Cagatay M, Petrowitsch T, et al. (2004) Acarbose reduces the risk for myocardial infarction in type 2 diabetic patients: meta-analysis of seven long-term studies. Eur Heart J 25, 10-16.

24. Esposito K, Giugliano D, Nappo F, et al. (2004) Regression of carotid atherosclerosis by control of postprandial hyperglycemia in type 2 diabetes mellitus. Circulation 110, 214-219.

25. Reaven GM (1988) Banting lecture 1988. Role of insulin resistance in human disease. Diabetes 37, 1595-1607.

26. Swislocki A, Chen Y, Golay A, et al. (1987) Insulin suppression of plasma-free fatty acid concentration in normal individuals and patients with type 2 (non-insulin-dependent) diabetes. Diabetologia 30, 622-626.

27. Ferrannini E, Barrett EJ, Bevilacqua S, et al. (1983) Effect of fatty acids on glucose production and utilization in man. J Clin Invest 72, 1737-1747.

28. Jorgensen RG, Russo L, Mattioli L, et al. (1988) Early detection of vascular dysfunction in type I diabetes. Diabetes 37, 292-296.

29. Marfella R, Verrazzo G, Acampora R, et al. (1995) Glutathione reverses systemic hemodynamic changes induced by acute hyperglycemia in healthy subjects. Am J Physiol 268, Suppl. 6, E1167-E1173.

30. Kawano H, Motoyama T, Hirashima O, et al. (1999) Hyperglycemia rapidly suppresses flow-mediated endotheliumdependent vasodilation of brachial artery. J Am Coll Cardiol 34, 146-154

31. Shige H, Ishikawa T, Suzukawa M, et al. (1999) Endothelium-dependent flow-mediated vasodilation in the postprandial state in type 2 diabetes mellitus. Am J Cardiol 84, 1272-1274.

32. Brownlee M (2001) Biochemistry and molecular cell biology of diabetic complications. Nature 414, 813-820.

33. Sakamoto T, Ogawa H, Kawano H, et al. (2000) Rapid change of platelet aggregability in acute hyperglycemia detection by a novel laser-light scattering method. Thromb Haemost 83, 475-479.

34. Ceriello A, Giacomello R, Stel G, et al. (1995) Hyperglycemia-induced thrombin formation in diabetes. The possible role of oxidative stress. Diabetes 44, 924-928.

35. Jones RL \& Peterson CM (1979) Reduced fibrinogen survival in diabetes mellitus. A reversible phenomenon. J Clin Invest 63, 485-493.

36. Nappo F, Esposito K, Cioffi M, et al. (2002) Postprandial endothelial activation in healthy subjects and in type 2 diabetic patients: role of fat and carbohydrate meals. $J \mathrm{Am}$ Coll Cardiol 39, 1145-1150. 
37. Esposito K, Nappo F, Marfella R, et al. (2002) Inflammatory cytokine concentrations are acutely increased by hyperglycemia in humans: role of oxidative stress. Circulation $\mathbf{1 0 6}$ 2067-2072.

38. Ceriello A, Falleti E, Motz E, et al. (1998) Hyperglycemiainduced circulating ICAM-1 increase in diabetes mellitus: the possible role of oxidative stress. Horm Metab Res 30, 146-149.

39. Diwadkar VA, Anderson JW, Bridges SR, et al. (1999) Postprandial low-density lipoproteins in type 2 diabetes are oxidized more extensively than fasting diabetes and control samples. Proc Soc Exp Biol Med 222, 178-184.

40. Ceriello A, Bortolotti N, Motz E, et al. (1999) Meal-induced oxidative stress and low-density lipoprotein oxidation in diabetes: the possible role of hyperglycemia. Metab Clin Exp 48, 1503-1508.

41. Wilson PW, D'Agostino RB, Levy D, et al. (1998) Prediction of coronary heart disease using risk factor categories. Circulation 97, 1837-1847.

42. Gotto AM (1998) Triglyceride as a risk factor for coronary artery disease. Am J Cardiol 82, 22-25.

43. Wood PD, Stefanick ML, Williams PT, et al. (1991) The effects on plasma lipoproteins of a prudent weight-reducing diet, with or without exercise, in overweight men and women. $N$ Engl J Med 325, 461-466.

44. Jeppesen J, Hein HO, Suadicani P, et al. (1998) Triglyceride concentration and ischemic heart disease: an eight-year follow-up in the Copenhagen Male Study. Circulation 97, 1029-1036.

45. Hokanson JE \& Austin MA (1996) Plasma triglyceride level is a risk factor for cardiovascular disease independent of highdensity lipoprotein cholesterol level: a meta-analysis of population-based prospective studies. J Cardiovasc Risk 3, 213-219.

46. Patsch JR, Miesenbock G, Hopferwieser T, et al. (1992) Relation of triglyceride metabolism and coronary artery disease. Studies in the postprandial state. Arterioscler Thromb 12, 1336-1345.

47. Peddie MC, Rehrer NJ \& Perry TL (2012) Physical activity and postprandial lipidemia: are energy expenditure and lipoprotein lipase activity the real modulators of the positive effect? Prog Lipid Res 51, 11-22.

48. Patsch JR, Karlin JB, Scott LW, et al. (1983) Inverse relationship between blood levels of high density lipoprotein subfraction 2 and magnitude of postprandial lipemia. Proc Natl Acad Sci U S A 80, 1449-1453.

49. Karpe F, Steiner G, Uffelman K, et al. (1994) Postprandial lipoproteins and progression of coronary atherosclerosis. Atherosclerosis 106, 83-97.

50. Bansal S, Buring JE, Rifai N, et al. (2007) Fasting compared with nonfasting triglycerides and risk of cardiovascular events in women. JAMA 298, 309-316.

51. Tabas I, Williams KJ \& Boren J (2007) Subendothelial lipoprotein retention as the initiating process in atherosclerosis: update and therapeutic implications. Circulation 116, 1832-1844.

52. Borén J, Matikainen N, Adiels M, et al. (2014) Postprandial hypertriglyceridemia as a coronary risk factor. Clin Chim Acta 431, 131-142.

53. Boquist S, Ruotolo G, Tang R, et al. (1999) Alimentary lipemia, postprandial triglyceride-rich lipoproteins, and common carotid intima-media thickness in healthy, middleaged men. Circulation 100, 723-728.

54. Teno S, Uto Y, Nagashima H, et al. (2000) Association of postprandial hypertriglyceridemia and carotid intima-media thickness in patients with type 2 diabetes. Diabetes Care 23, 1401-1406.
55. van Oostrom AJ, Sijmonsma TP, Verseyden C, et al. (2003) Postprandial recruitment of neutrophils may contribute to endothelial dysfunction. J Lipid Res 44, 576-583.

56. Van Wijk J, Cabezas MC, Coll B, et al. (2006) Effects of rosiglitazone on postprandial leukocytes and cytokines in type 2 diabetes. Atherosclerosis 186, 152-159.

57. Vogel RA, Corretti MC \& Plotnick GD (1997) Effect of a single high-fat meal on endothelial function in healthy subjects. Am J Cardiol 79, 350-354.

58. Erridge C, Attina T, Spickett CM, et al. (2007) A high-fat meal induces low-grade endotoxemia: evidence of a novel mechanism of postprandial inflammation. Am J Clin Nutr 86, 1286-1292.

59. Wildman RE \& Medeiros DM (1999) Advanced Human Nutrition. Boca Raton, FL: CRC Press.

60. Czech MP \& Corvera S (1999) Signaling mechanisms that regulate glucose transport. J Biol Chem 274, 1865-1868.

61. Mashek DG \& Coleman RA (2006) Cellular fatty acid uptake: the contribution of metabolism. Curr Opin Lipidol 17, 274-278.

62. Haag M \& Dippenaar NG (2005) Dietary fats, fatty acids and insulin resistance: short review of a multifaceted connection. Med Sci Monit 11, RA359-RA367.

63. Hulbert AJ, Turner N, Storlien L, et al. (2005) Dietary fats and membrane function: implications for metabolism and disease. Biol Rev Camb Philos Soc 80, 155-169.

64. Randle P, Garland P, Hales C, et al. (1963) The glucose fattyacid cycle its role in insulin sensitivity and the metabolic disturbances of diabetes mellitus. Lancet 281, 785-789.

65. Storlien LH, Jenkins AB, Chisholm DJ, et al. (1991) Influence of dietary fat composition on development of insulin resistance in rats: relationship to muscle triglyceride and $\omega$-3 fatty acids in muscle phospholipid. Diabetes 40, 280-289.

66. Lovejoy JC (1999) Dietary fatty acids and insulin resistance. Curr Atheroscler Rep 1, 215-220.

67. Fernie AR, Carrari F \& Sweetlove LJ (2004) Respiratory metabolism: glycolysis, the TCA cycle and mitochondrial electron transport. Curr Opin Plant Biol 7, 254-261.

68. Lunt SY \& Vander Heiden MG (2011) Aerobic glycolysis: meeting the metabolic requirements of cell proliferation. Annu Rev Cell Dev Biol 27, 441-464.

69. Ceriello A \& Motz E (2004) Is oxidative stress the pathogenic mechanism underlying insulin resistance, diabetes, and cardiovascular disease? The common soil hypothesis revisited. Arterioscler Thromb Vasc Biol 24, 816-823.

70. Vincent AM, McLean LL, Backus C, et al. (2005) Short-term hyperglycemia produces oxidative damage and apoptosis in neurons. FASEB J 19, 638-640.

71. Jay D, Hitomi H \& Griendling KK (2006) Oxidative stress and diabetic cardiovascular complications. Free Radic Biol Med 40, 183-192.

72. Rocha VZ \& Libby P (2009) Obesity, inflammation, and atherosclerosis. Nat Rev Cardiol 6, 399-409.

73. Hansson GK (2005) Inflammation, atherosclerosis, and coronary artery disease. $N$ Engl J Med 352, 1685-1695.

74. Kita T, Kume N, Ishii K, et al. (1999) Oxidized LDL and expression of monocyte adhesion molecules. Diabetes Res Clin Pract 45, 123-126.

75. Hajjar DP \& Haberland ME (1997) Lipoprotein trafficking in vascular cells. Molecular Trojan horses and cellular saboteurs. J Biol Chem 272, 22975-22978.

76. Maziere C, Auclair M, Djavaheri-Mergny M, et al. (1996) Oxidized low density lipoprotein induces activation of the transcription factor NFKB in fibroblasts, endothelial and smooth muscle cells. IUBMB Life 39, 1201-1207.

77. Weissman A, Lowenstein L, Peleg A, et al. (2006) Power spectral analysis of heart rate variability during the 100-g oral 
glucose tolerance test in pregnant women. Diabetes Care 29, 571-574.

78. Ferreira AC, Peter AA, Mendez AJ, et al. (2004) Postprandial hypertriglyceridemia increases circulating levels of endothelial cell microparticles. Circulation 110, 3599-3603.

79. Emerson SR, Kurti SP, Snyder BS, et al. (2016) Effects of thirty and sixty minutes of moderate-intensity aerobic exercise on postprandial lipemia and inflammation in overweight men: a randomized cross-over study. J Int Soc Sports Nutr 13, 1.

80. Albrink MJ, Fitzgerald JR \& Man EB (1958) Reduction of alimentary lipemia by glucose. Metabolism 7, 162-171.

81. Mann J, Truswell A \& Pimstone B (1971) The different effects of oral sucrose and glucose on alimentary lipaemia. Clin Sci 41, 123-129.

82. Cohen JC \& Schall R (1988) Reassessing the effects of simple carbohydrates on the serum triglyceride responses to fat meals. Am J Clin Nutr 48, 1031-1034.

83. Knuth ND, Remias DB \& Horowitz JF (2008) Adding carbohydrate to a high-fat meal blunts postprandial lipemia in women and reduces meal-derived fatty acids in systemic circulation. Appl Physiol Nutr Metab 33, 315-325.

84. Westphal S, Leodolter A, Kahl S, et al. (2002) Addition of glucose to a fatty meal delays chylomicrons and suppresses VLDL in healthy subjects. Eur J Clin Invest 32, 322-327.

85. Collier G \& O'Dea K (1983) The effect of coingestion of fat on the glucose, insulin, and gastric inhibitory polypeptide responses to carbohydrate and protein. Am J Clin Nutr 37, 941-944.

86. Ercan N, Gannon MC \& Nuttall FQ (1994) Effect of added fat on the plasma glucose and insulin response to ingested potato given in various combinations as two meals in normal individuals. Diabetes Care 17, 1453-1459.

87. Chong MF, Fielding BA \& Frayn KN (2007) Mechanisms for the acute effect of fructose on postprandial lipemia. $A m J$ Clin Nutr 85, 1511-1520.

88. Langsted A, Freiberg JJ \& Nordestgaard BG (2008) Fasting and nonfasting lipid levels: influence of normal food intake on lipids, lipoproteins, apolipoproteins, and cardiovascular risk prediction. Circulation 118, 2047-2056

89. Mihas C, Kolovou GD, Mikhailidis DP, et al. (2011) Diagnostic value of postprandial triglyceride testing in healthy subjects: a meta-analysis. Curr Vasc Pharmacol 9, 271-280.
90. Weiss EP, Fields DA, Mittendorfer B, et al. (2008) Reproducibility of postprandial lipemia tests and validity of an abbreviated 4-hour test. Metab Clin Exp 57, 1479-1485.

91. Kolovou GD, Mikhailidis DP, Kovar J, et al. (2011) Assessment and clinical relevance of non-fasting and postprandial triglycerides: an expert panel statement. Curr Vasc Pharmacol 9, 258-270.

92. Delgado-Lista J, Perez-Jimenez F, Ruano J, et al. (2010) Effects of variations in the APOA1/C3/A4/A5 gene cluster on different parameters of postprandial lipid metabolism in healthy young men. J Lipid Res 51, 63-73.

93. Perez-Martinez P, Lopez-Miranda J, Perez-Jimenez F, et al. (2008) Influence of genetic factors in the modulation of postprandial lipemia. Atheroscler Suppl 9, 49-55.

94. López-Miranda J, Cruz G, Gómez P, et al. (2004) The influence of lipoprotein lipase gene variation on postprandial lipoprotein metabolism. J Clin Endocrinol Metab 89, 4721-4728.

95. Jiménez-Gómez Y, Pérez-Jiménez F, Marín C, et al. (2008) The - 250G/A polymorphism in the hepatic lipase gene promoter influences the postprandial lipemic response in healthy men. Nutr Metab Cardiovasc Dis 18, 173-181.

96. Fumeron F, Aubert R, Siddiq A, et al. (2004) Adiponectin gene polymorphisms and adiponectin levels are independently associated with the development of hyperglycemia during a 3 -year period: the epidemiologic data on the insulin resistance syndrome prospective study. Diabetes 53, 1150-1157.

97. Hara K, Boutin P, Mori Y, et al. (2002) Genetic variation in the gene encoding adiponectin is associated with an increased risk of type 2 diabetes in the Japanese population. Diabetes 51, 536-540.

98. Salpea KD, Talmud PJ, Cooper JA, et al. (2010) Association of telomere length with type 2 diabetes, oxidative stress and UCP2 gene variation. Atherosclerosis 209, 42-50.

99. Cauchi S, Meyre D, Choquet H, et al. (2006) TCF7L2 variation predicts hyperglycemia incidence in a French general population: the data from an epidemiological study on the Insulin Resistance Syndrome (DESIR) study. Diabetes $\mathbf{5 5}$, 3189-3192.

100. Vaquero AR, Ferreira NE, Omae SV, et al. (2012) Using genenetwork landscape to dissect genotype effects of TCF7L2 genetic variant on diabetes and cardiovascular risk. Physiol Genomics 44, 903-914. 